\title{
Research on Technologies and Applications of Embedded Internet
}

\author{
BingJie Liu,Jianhong Ren \\ Jiangxi Vocational and Technical College of Communication, Jiangxi, Nanchang 330013, China
}

Keywords: Technologies and Applications; Embedded Internet; TCP/IP

Abstract. As the appliance of embedded internet is more and more abroad, the embedded internet technologies have become the focus of the electronics research in the world. This paper discusses the concept and key technologies of the embedded internet, and presents the actual applications of the embedded internet technologies in recent years to provide some references for the relevant researchers.

\section{Concept of Embedded Internet}

Embedded internet is a differentiated computing platform. It is "invisible" to the users and has a predetermined function. Compared with the personal computer, embedded devices need to put the limited computing power in a main application. Embedded technology is very suitable for the development trend and characteristics of the terminal market. Embedded platform is generally for some specific applications and development. It is easy to use and easy to upgrade. After the stages of "mainframe", server and PC "," mobile phones and mobile Internet devices (MID) ", we call the embedded devices as the carrier "embedded Internet". In the upcoming fourth stage, embedded devices and applications will really get to the Internet everywhere, people both in work, entertainment, learning and rest time, time and the Internet to remain connected. ITU will the development of the Internet is divided into four stages. The fourth stage is full of all kinds of professional equipment, including household appliances, medical equipment, and industrial machinery. It marked the arrival of the embedded Internet era.

\section{Technologies of Embedded Internet}

Embedded internet technology is to realize the combination of electronic equipment and internet with microprocessor. The ideas of the combination of embedded system and internet emerged very early. The main difficulty lies in the internet communication protocol requirements for computer memory and computing speed is relatively high, and embedded systems except some 32-bit processor. The existence of a large number of is 8-bit and 16 bit MCU can support TCP/IP Internet protocol which will occupy a large amount of system resources. At present, the technologies of embedded internet can be roughly divided into the following two categories:

Integration Technology of TCP/IP. We adopt the processor and TCP/IP agreement technology when the MCU processor acts as a personal computer. It normally takes high-end processor, such as 32-bit arm, SH3, MIPs MCU and some single cycle instruction high speed 8-bit MCU, such as AVR and SX. The structure is shown in Fig. 1: 


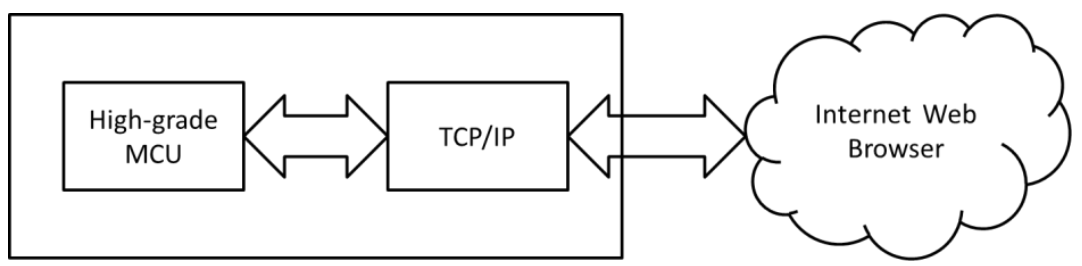

Fig. 1. Structure of the Integration Technology of TCP/IP

There are two ways to deal with the TCP/IP protocol. One way is to use the real-time operating system RTOS, using the software to deal directly with the TCP/IP protocol. The function of the real time operating system is more and more powerful, and many of them have the ability to support the image interface and TCP/IP. The most flexible way, can realize many complex functions according to the requirement of users and of course flexible at the same time bring is development complexity increases, high requirements for developers, the operating system and TCP/IP protocol to have a certain familiarity. So the development period is long, the price is very high. The other is the use of the TCP/IP protocol solidified the hardware chip, which supports HTTP, SMTP, MME, POP3 and other protocols, through the external hardware circuit to deal with the TCP/IP protocol. So the execution speed is up to IOOMIPS. SX MCU's biggest feature is support for virtual peripherals. Many function modules are designed into a software function module and need a direct calls to the corresponding module, the effect equivalent to install the corresponding peripheral hardware circuit, but the virtual peripheral way more flexible and convenient, and the hardware circuit is simple. Compared with the former one, the method is more convenient and the development of the difficulty is reduced. However, it needs that the developers are familiar with the TCP/IP protocol and the related interface.

Connection Technology of Webit. Webit is a practical product of embedded system connecting to the internet. It links the MCU and Ethernet controller into a boss card, making the embedded system complete the connection of embedded system and Internet network. Webit has its own IP address, and mentioned the first way is similar, but it has a high degree of integration, the protocol processing part is independent, developers omitted part of the network design, will focus on the application of the system itself. The webit in general is a system based on AVR microcontroller, in the singlechip with EEPROM storage system service program of 8 Kbytes of flash space, 512 bytes of RAM space for a and used to store the system parameters (IP address, MAC address, baud rate, etc.). In the periphery of the microcontroller, there is used to store the EEPROM page of the Web. The system contains an IOM Ethernet controller, which is used to provide network connection. The system provides the application system interface for the UART level of the TTL port and the 14 bit I/O port, and its application is shown in Fig. 2:

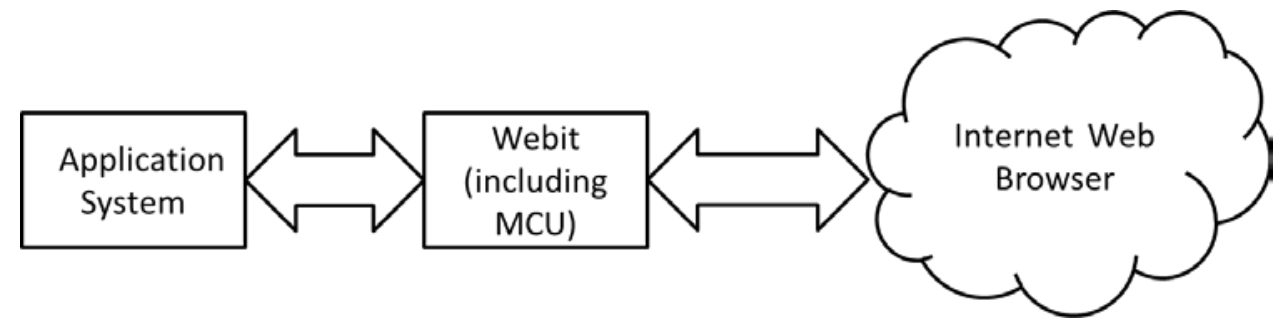

Fig. 2. Application of Connection Technology of Webit

Webit technology is actually similar to the first way, but it will be separated from the protocol processing part of the Webit alone. The developers do not have to consider the network protocol and related interfaces, but also need to be independent of the IP address. The method does not need 
a gateway. The structure of the software and the hardware can be independently designed, and the development cost is relatively low.

\section{Applications of Embedded Internet}

Visual Phone. At present, many countries, including China, have adopted H.323 as the protocol between the IP phone gateways. In fact, the IP phone gateways are used as the interfaces between the circuit switching network and the IP network. However, in the next generation network, due to the application of a large number of IP products, the end to end must be based on pure IP network. 3GPP has identified the SIP protocol as the third generation mobile communication all IP network control protocol. With the development of these standards and the further increase of network bandwidth, the network video phone based on SIP in the pure IP network will be more and more widely used. Both H.323 and SIP are the communication protocols for the realization of IP voice technology and multimedia applications. Network telephone communication with its low cost of this unparalleled advantage further exacerbated the development of pure IP network. In the technical aspects of the development of domestic and international telecommunications industry is basically synchronous, many telecom equipment manufacturers launched SIP based products and services. China has the world's largest VoIP network, the major operators have been aware of the huge advantage of SIP, have carried out SIP network experiment. SIP is an independent protocol, and easy to use. SIP protocol is a hierarchical, generally divided into three layers. It uses a BNF grammar rules to; the second layer to the transport layer, the layer defines the client how to send requests and receive responses and server how to receive a request and sends the response; the third layer for transaction layer, the layer application layer retransmission and matching ring to request, and handle the application layer timeout. The user layer users, users use the keyboard to dial, answer and hang up operation. The interface layer completes two functions, one is the application layer and the transport layer interface - establishing socket; two is to have the calling module each module interface function layer. In encoding, the input data is sampled by $8 \mathrm{kHz} 16$ bit linear PCM speech signal, frame length is $30 \mathrm{~ms}$. Based on the original speech analysis, get the relevant parameters and transfer encoding. In the decoder, we use these parameters to construct the excitation signal and the synthesis filter. The excitation signal can be obtained by the original speech synthesis filter.

Video Development. The rise of the Interactive Protocol Television (IPTV) can provide viewers with a new way to choose and control their broadcast content. Domestic and foreign operators have invested in the construction of their own IPTV operating platform. Viewers not only want to be able to receive high-quality IPTV content, but also want to be able to watch as ordinary TV. With the development of IPv6 network, the bandwidth is more and more abundant, and the development of embedded technology provides the basis for the convenience of the content. Based on this reality, a home set-top box through play Internet video application architecture, solve play these Internet video must through the computer problems, the watch the video content becomes simple and easy to use. Typical Internet video sites include several basic components: video introduction, video classification, video itself. Before we play the selected video through a browser or special software, we must first find the corresponding video by keyword search or classification search, and then we can play. Most of the video sites are using streaming media playback mode, allows us to download and play, a few need to download to play. We design the block using the embedded operating system is Linux, has the following characteristics: open source code, small kernel, high efficiency, good support for IPv6; Linux height module of make it easier for loading and unloading component; built-in network support and is easy to realize the built-in web server; support multiple hardware 
platforms. It provides complete development tool and SDK, including the graphical customization and configuration tools. When the embedded network video is on demand, it is necessary to carry out real-time image processing, audio and video decoding and synchronization, network transmission, graphical user interface, the four part of the collaborative processing. In order to ensure that the software development, integration, and the final test are not too complicated, we have to deal with the four parts. Alarm in the deployment of state and correlate alarms and alarm equipment in case of alarm, the alarm will immediately in alarm state, which also triggers the alarm linkage. Usually, the alarm is in normal state. Once the corresponding equipment alarms, the alarm is in the deployment of state. The alarm will turn to the normal state until the release of it.

\section{Conclusion}

With the development and progress of computer technology, the embedded internet has been widely used, including network phone, video development, intelligent instrument, industrial detection system and security system and so on. Embedded technology and internet technology can effectively unify in together. The embedded system development space will be greatly expanded in the future.

\section{References}

[1] Miao Cencen, The application of embedded internet in the network home appliance [J]. China Appliance Technology, 2014(9): 66-68.

[2] Gao Changyan, Research and the realization on the embedded TCP/IP protocol [D]. University of Chinese Academy of Sciences, 2005

[3] Liu Chunmei, Hu Jianliang, Jiang Jingmei, Internet Data and Broadcast Data Processing Method Based on Embedded Browser [J]. Journal of Network New Media, 2014, 3(2): 32-37.

[4] Zhang Yonglai, Liu Jianhui, Jiang Liying, Jiang Liqun Analysis and Implementation of Embedded Internet Visual Phone Based on SIP [J]. Computer and Information Technology, 2007, 15(5): 63-65.

[5] WANG Xiao-Hua, LI Ping. Embedded Internet System Based on WIFI/GPRS[J]. Computer Systems \& Applications, 2014. 